\title{
A Novel Combined Fused Silica Cylinder Shell Vibrating Gyroscope
}

\author{
Xiang Xi, Yulie $\mathrm{Wu}^{*}$, Xiaomei Wu, Qinglei Luan and Xuezhong Wu \\ Department of Mechatronics Engineering, National University of Defense Technology, Hunan, \\ Changsha 410073, P. R. China
}

(Received August 27, 2012; accepted December 20, 2012)

Key words: cylinder shell vibrating gyroscope, combined resonator, fused silica, bias stability

We propose a novel combined fused silica cylinder shell vibrating gyroscope. The cylinder wall and the bottom disk of the fused silica resonator are fabricated separately and then are connected by glue. By using this method, the difficulty of manufacturing the fused silica cylinder vibrating gyroscope can be significantly reduced. The structure characteristics and vibration modes of the combined fused silica resonator are first analyzed and then the fabrication processes of the gyroscope are introduced, including the machining process of the combined resonator, the mass balancing method for minimizing the natural frequency split, and the control circuit of the gyroscope. Finally, a performance test of the combined gyroscope was implemented, including a $\mathrm{Q}$ factor test, frequency stability test, and bias stability test. Experimental results show that the combined fused silica cylinder vibrating gyroscope has good bias stability at room temperature and has the potential to achieve higher accuracy.

\section{Introduction}

The cylinder shell vibrating gyroscope is a type of solid-state vibrating gyroscope with a symmetrical structure. ${ }^{(1)}$ Its counterparts such as hemispherical resonator gyroscopes, disk gyroscopes, and vibrating ring gyroscopes are well known for their high accuracy, low power consumption, long life, and so on. ${ }^{(2)}$ The cylinder shell vibrating gyroscope has the additional advantages of a simple manufacturing process and low cost. $^{(3)}$ These characteristics have already caught the attention of many researchers. Cylinder shell vibrating gyroscopes made of different materials have been reported, such as piezoelectric gyroscopes and metallic gyroscopes. ${ }^{(3,4)}$ These materials are easy to machine, but their stability is unsatisfactory.

*Corresponding author: e-mail: ylwu_nudt@sina.com 
In order to improve the performance of the gyroscope, fused silica can be employed as the resonator material of the cylinder shell vibrating gyroscope. Compared with the metallic, piezoelectric, quartzose, or silicic material commonly used in gyroscope fabrication, fused silica has the advantages of a high $\mathrm{Q}$ factor and good temperature stability.(5) Usually, circular gyroscopes are fabricated by micro-electromechanical systems (MEMS) technology, such as the spoke gyroscope reported by Sung et al..$^{(5)}$ and the high-aspect-ratio combined poly and single-crystal silicon (HARPSS) polysilicon vibrating ring gyroscope reported by Ayazi and Najafi. ${ }^{(6)}$ These gyroscopes have relatively small sizes, but the accuracy is limited by the small output signals of the vibratory structure. ${ }^{(6,7)}$ Watson designed a vibrating gyroscope made of a piezoelectric material, but its stability is also limited due to the inhomogeneity of the material. ${ }^{(4)}$ In many cases, circular shell gyroscopes made of fused silica show better performance. Hemispherical resonator gyroscopes can reach an accuracy of $10^{-4} \% / \mathrm{h} \cdot{ }^{(8)}$ A $10 \mathrm{~mm}$ cylinder gyroscope can also approach an accuracy of $1 \%$ h or possibly better. ${ }^{(9)}$ However, fused silica is difficult to machine due to its brittleness. For a cylinder shell vibrating gyroscope, some parts of the resonator are very thin (less than $0.5 \mathrm{~mm}$ ). The machining stress, especially the stress acting during the turning of the structure, may easily break the shell structure. Therefore, a monolithic fused silica cylinder shell resonator is extremely difficult to machine with high precision by the conventional way. Moreover, the complicated manufacturing process of the monolithic fused silica resonator can lead to a low yield. In view of this, a novel fused silica resonator based on a combined structure is proposed in this paper, which will greatly reduce the difficulty of manufacturing the fused silica resonator.

In this paper, we investigate the possibility of developing a novel combined gyroscope, and present the simulation, fabrication, and experimental evaluation of the novel gyroscope. The operation principle of the cylinder shell vibrating gyroscope is introduced in $\S 2$. The structure characteristics and temperature performance of the combined fused silica resonator are analyzed in $\S 3$. The fabrication processes of the combined fused silica cylinder shell vibrating gyroscope are described in $\S 4$, including the machining process of the fused silica resonator, the mass balancing method of minimizing the natural frequency split, and the control circuit of the gyroscope. Practical test experiments on a prototypal gyroscope are reported in $\S 5$, including a Q factor test frequency stability test and bias stability test. Finally, conclusions are made in $\S 6$.

\section{Operation Principle of the Cylinder Shell Vibrating Gyroscope}

The basic structure of the cylinder shell vibrating gyroscope, which comprises a cylinder wall, a circular bottom disk, and a substrate, is presented in Fig. 1. The driving and sensing piezoelectric electrodes are attached to the bottom by gluing. The piezoelectric material PZT-5 can be used for gluing the electrode due to its high sensitivity and good stability. In order to obtain a strong electrical signal, the electrodes should be designed to utilize as much of the area of the bottom as possible. It is clear that the main vibratory structure element is the cylinder wall; thus, the contribution of the piezoelectric electrodes to the thermal stability is small and can be compensated by the circuit. ${ }^{(10,11)}$ 

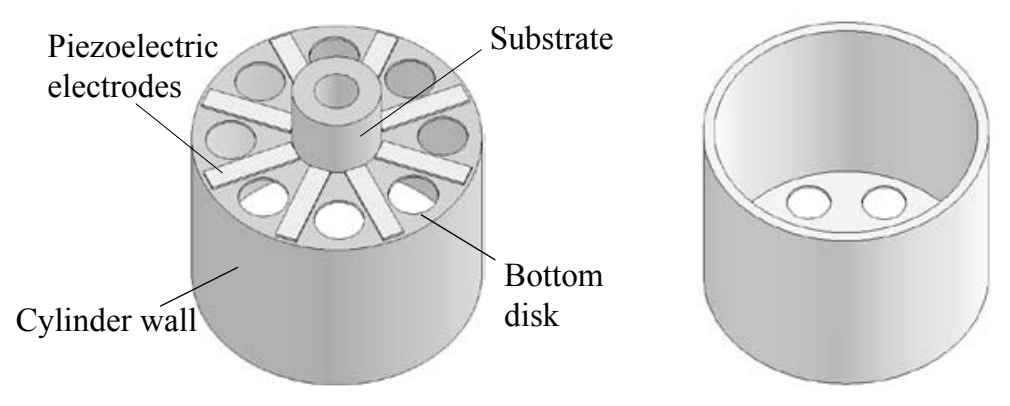

Fig. 1. Structure of the cylinder shell vibrating gyroscope.

The operation principle of the cylinder shell vibrating gyroscope is illustrated in Fig. 2. When an alternating voltage is applied on the two piezoelectric electrodes attached to the spokes in the $X$ axis, the piezoelectric electrodes expand and contract owing to the converse piezoelectric effect, which can produce an alternating bending moment on the spokes. Therefore, the driving mode is excited along the $X$ and $Y$ directions. When the resonator rotates at an angular velocity $\omega$, the sensing mode appears in the $X^{\prime}$ and $Y^{\prime}$ directions due to Coriolis force vector $F_{\mathrm{c}}$. In the sensing mode, the spokes vibrate in the $X^{\prime}$ and $Y^{\prime}$ directions and the piezoelectric electrodes attached to them contract and expand alternately. The strain of the piezoelectric electrodes generates an output voltage to produce a piezoelectric effect, which is proportional to the angular velocity $\omega$ and can be detected by a readout circuit. ${ }^{(12,13)}$

\section{Structure and Finite Element (FE) Modeling}

\subsection{Description of combined structure}

As shown in Fig. 3, the combined fused silica resonator comprises two parts: the cylinder wall of the resonator and the bottom disk. The circular holes in the bottom disk are used to decrease the interaction of the electrodes; hence, the vibration coupling between the driving mode and sensing mode is decreased. Simultaneously, the energy loss in the bottom disk can be decreased, and the sensitivity of the gyroscope is thus improved. The two parts are fabricated separately, and then are connected by a special glue. For a medium-size gyroscope, the fused silica cylinder wall of the resonator can be machined by precision grinding, and the fused silica bottom disk with holes can be machined by laser cutting.

\subsection{FE modeling}

In order to obtain the appropriate operating frequency and modal shape, the sizes of the resonator structure are designed by using the FE method. The FE model of the combined fused silica resonator is constructed using ANSYS software. Considering that the glue is solid and rigid after drying, SOLID95 elements are used to construct 


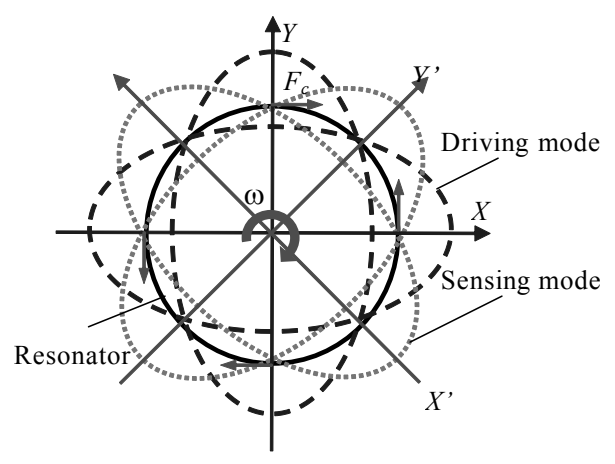

Fig. 2. Schematic of the operation principle.

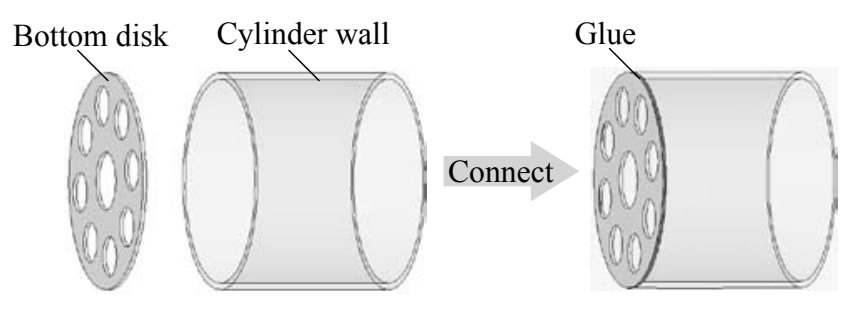

Fig. 3. Combined fused silica resonator.

the glue layer model. The material parameters used in the model are listed in Table 1. The operation frequency of the resonator should be higher than most frequencies of environmental disturbances, which are below $2000 \mathrm{~Hz}$, and also should be far from the frequency of the adjacent eigenmodes. Simulation results show that the properties of the glue only slightly influence the eigenfrequencies of the resonator (less than $5 \mathrm{~Hz}$ variation when the elasticity modulus of the glue ranges from 1 to $100 \mathrm{Gpa}$ ). Therefore, the eigenfrequencies are mainly determined by the size of the resonator. The basic structure parameters of the combined fused silica resonator are given in Table 2.

The results of modal analysis are shown in Fig. 4. The active mode frequency is $3643 \mathrm{~Hz}$, which is coincident with the sensing mode. The equivalent frequency of the active mode and sensing mode enables the Coriolis force to induce a sensing mode with high sensitivity. ${ }^{(13)}$ The modal results for the adjacent eigenmodes are also given in Fig 4. The first-order mode indicates the swing of the resonator, and its frequency is $521 \mathrm{~Hz}$. The second-order mode indicates the axial motion of the resonator, and its frequency is $564 \mathrm{~Hz}$. The fifth-order mode frequency is $9798 \mathrm{~Hz}$. 
Table 1

Material parameters of the resonator.

\begin{tabular}{lc}
\hline Description (unit) & Value \\
\hline Young's modulus of fused silica $(\mathrm{GPa})$ & 77.8 \\
Density of fused silica $\left(\mathrm{kg} / \mathrm{m}^{3}\right)$ & 2210 \\
Poisson's ratio of fused silica & 0.17 \\
Thermal expansion coefficient & $5 \times 10^{-7}$ \\
\hline
\end{tabular}

Table 2

Geometric parameters of the resonator.

\begin{tabular}{ll}
\hline Description (unit) & Value \\
\hline Outer diameter of cylinder wall $(\mathrm{mm})$ & 30 \\
Height of cylinder wall $(\mathrm{mm})$ & 25 \\
Thickness of cylinder wall $(\mathrm{mm})$ & 0.9 \\
Thickness of bottom disk $(\mathrm{mm})$ & 0.5 \\
Diameter of holes $(\mathrm{mm})$ & 5.5 \\
\hline
\end{tabular}

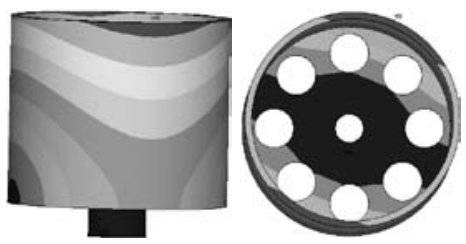

(a)

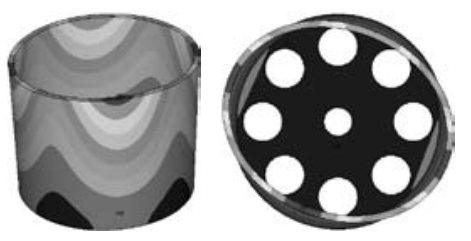

(d)

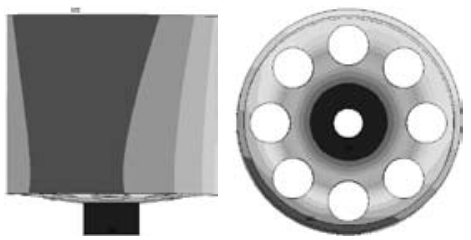

(b)

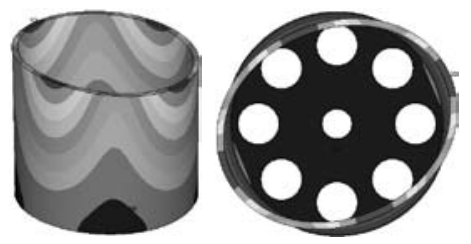

(c)

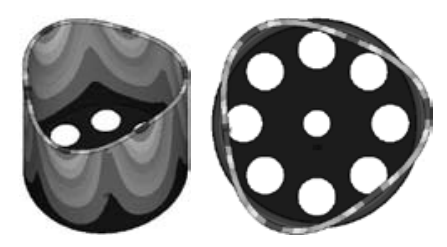

(e)

Fig. 4. Mode contours. (a) First-order mode, (b) second-order mode, (c) driving mode, (d) sensing mode, and (e) fifth-order mode.

\subsection{Analysis and comparison}

It is well known that one of the main advantages of fused silica is its good temperature stability. However, for the combined structural gyroscope, a mismatch of thermal expansion ratios is induced due to the use of the glue and the piezoelectric electrodes. When thermal deformation occurs, bending of the bottom disk can be observed, as 
shown in Fig. 5. Based on the operation principle of the cylindrical gyroscope, there should be no signal outputs at the nodal positions when there is no angular velocity input. However, when the bending deformation happens, the piezoelectric electrodes generates a biased voltage signal owing to a piezoelectric effect. This voltage directly contributes to the bias stability of the gyroscope since the temperature is variable in practice.

Since the properties of the curing glue are important for the combined structure, the influence of the curing glue on the gyroscope should be determined first. We set the reference temperature as $0{ }^{\circ} \mathrm{C}$ in the simulation, and then change the environmental temperature to a certain value $\left(60^{\circ} \mathrm{C}\right.$ in this study).(14) $\mathrm{By}$ changing the parameter values of the glue, thermal deformation graphs of the bottom disk can be obtained, as shown in Fig. 6. It can be seen that when the elastic modulus of the glue is increased from 1 to $100 \mathrm{Gpa}$ (with the thermal expansion coefficient maintained at 0.001 and a thickness of $5 \mu \mathrm{m}$ ), the deformation of the bottom disk increases from 0.46 to $0.77 \mu \mathrm{m}$. When the thermal expansion coefficient of the glue is increased from 0.001 to 0.01 (with the elastic modulus maintained at $1 \mathrm{Gpa}$ and a thickness of $5 \mu \mathrm{m}$ ), the deformation of the bottom disk increases from 0.46 to $1.50 \mu \mathrm{m}$. Figure 6 also reveals that a thicker glue may increase the deformation. When the thickness of the glue is increased from 1 to $10 \mu \mathrm{m}$ (with the elastic modulus maintained at $1 \mathrm{Gpa}$ and a thermal expansion coefficient of 0.001 ), the deformation of the bottom disk increases from 0.26 to $0.70 \mu \mathrm{m}$. Thus, glue with a small elastic modulus, low thermal expansion coefficient, and low thickness can reduce the thermal deformation of the resonator.

After the parameters of the combined structure are determined, the thermal performance of the gyroscope can be analyzed on the basis of the previous model. The steps for the thermal analysis are as follows.

First, thermal stress analysis is conducted to obtain the stress magnitude and thermal deformation under different temperatures. Secondly, modal analysis is performed including the thermal stress generated in the first step to obtain the eigenfrequencies. Then, vibration analysis is implemented by using the mode superposition method. For convenience, a pair of driving forces $(0.001 \mathrm{~N})$ is used to imitate the effect of the driving electrodes. Thus, a quantitative analysis of the temperature performance can be acquired
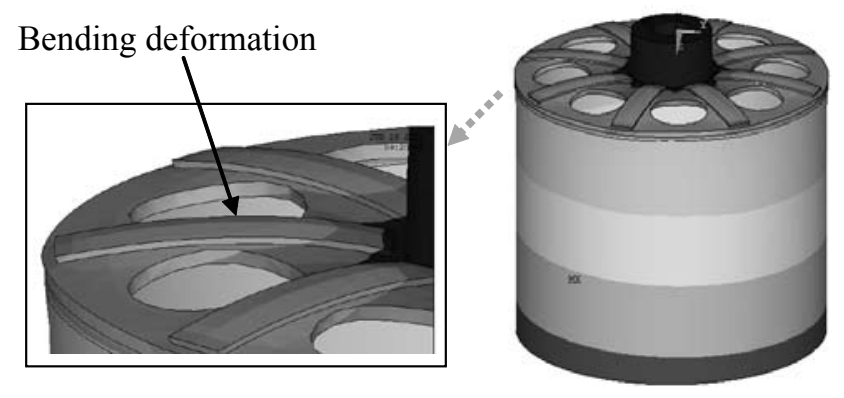

Fig. 5. Thermal deformation of the resonator. 


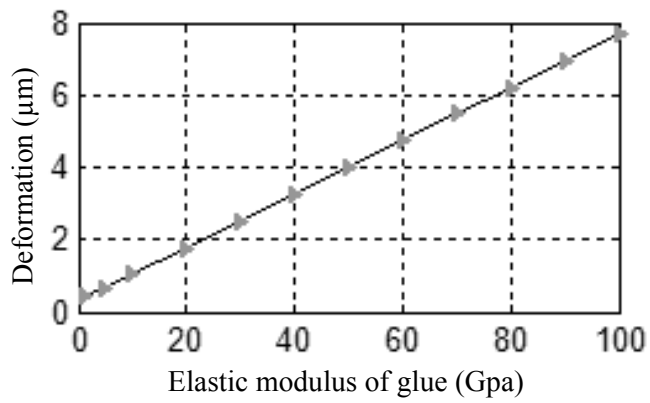

(a)

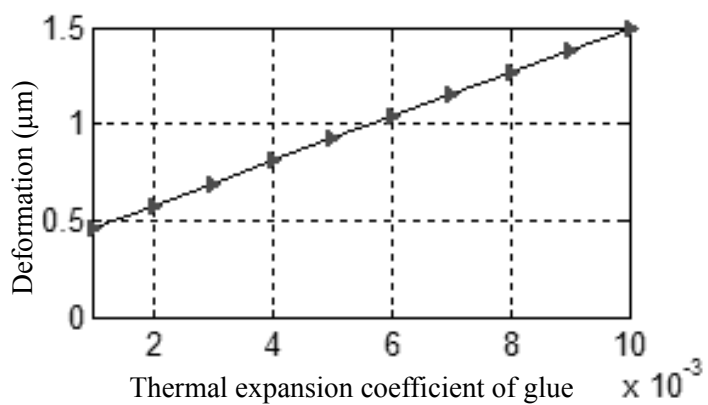

(b)

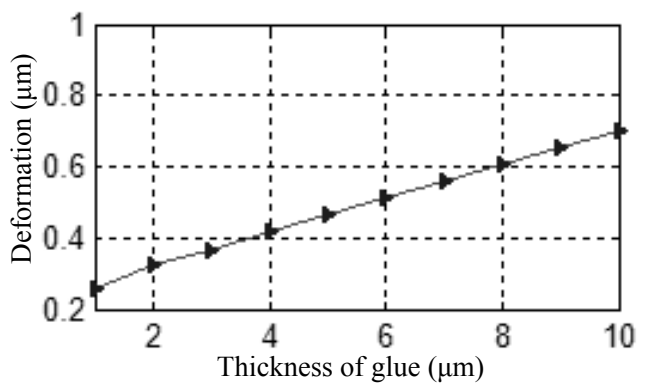

(c)

Fig. 6. Deformation of the bottom disk due to temperature variation.

by following the above steps. The reference temperature in the simulation is $0{ }^{\circ} \mathrm{C}$, and the environmental temperature ranges from -40 to $60^{\circ} \mathrm{C}$.

For the sake of comparison, four different cylinder shell vibrating gyroscopes are considered. The geometric parameters of the compared gyroscopes are the same, but the materials of the resonators are different. The influence of piezoelectric electrodes is also considered in the analysis. The different cases are listed as follows:

(1) Case 1: a monolithic gyroscope without gluing the piezoelectric electrodes (the resonator material is fused silica, and its parameters are given in Table 1).

(2) Case 2: a monolithic gyroscope with piezoelectric electrodes attached on the bottom disk (the resonator material is fused silica, and the physical parameters of the piezoelectric electrodes are a Young's modulus of $110 \mathrm{Gpa}$, a mass density of 7500 $\mathrm{kg} / \mathrm{m}^{3}$, a Poisson's ratio of 0.27 , and a thermal expansion coefficient of $\left.4 \times 10^{-6}\right)$. $^{(15)}$

(3) Case 3: a monolithic gyroscope with piezoelectric electrodes attached on the bottom disk (the resonator material is a nickel alloy with a Young's modulus of $210 \mathrm{Gpa}$, a mass density of $7850 \mathrm{~kg} / \mathrm{m}^{3}$, a Poisson's ratio of 0.28 , and a thermal expansion coefficient of $\left.1 \times 10^{-4}\right)$.

(4) Case 4: a combined fused silica gyroscope with piezoelectric electrodes attached on the bottom disk. 
The simulation results are shown in Fig. 7. The monolithic gyroscope without gluing the piezoelectric electrodes possesses the smallest thermal deformation (bending deformation of the bottom disk). After the piezoelectric electrodes are glued on the bottom disk, the deformation becomes larger due to the mismatch of thermal expansion ratios. For the combined fused silica gyroscope, its structural deformation is larger than that of a single body. The deformation magnitudes for gyroscopes (1), (2), and (4) are of the same order. However, the gyroscope made of nickel alloy shows significantly larger deformation. It should be noted that the amplitude of the resonator may also be affected by varying the temperature, as shown in Fig. 8 . However, the order of magnitude of the amplitude variation is much smaller than that of the static thermal deformation. Thus, the temperature drift of these gyroscopes is mainly caused by the thermal deformation of the structure. Therefore, the thermal performance of the combined gyroscope is slightly lower than that of a monolithic gyroscope but far better than that of a metallic gyroscope.

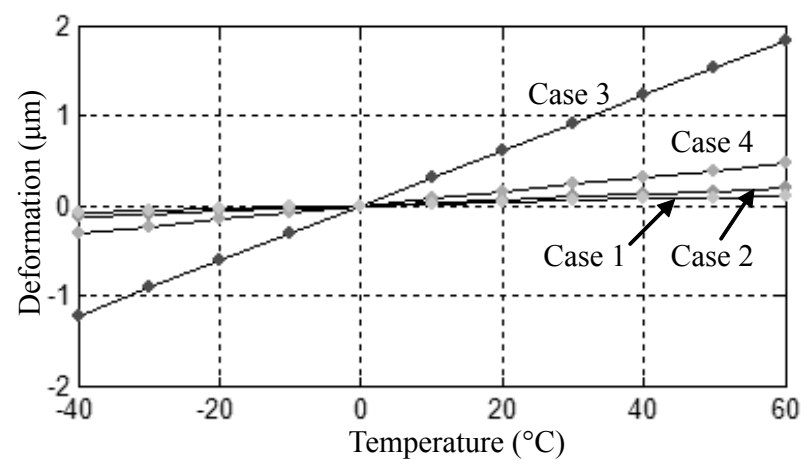

Fig. 7. Deformation of resonators due to temperature variation.

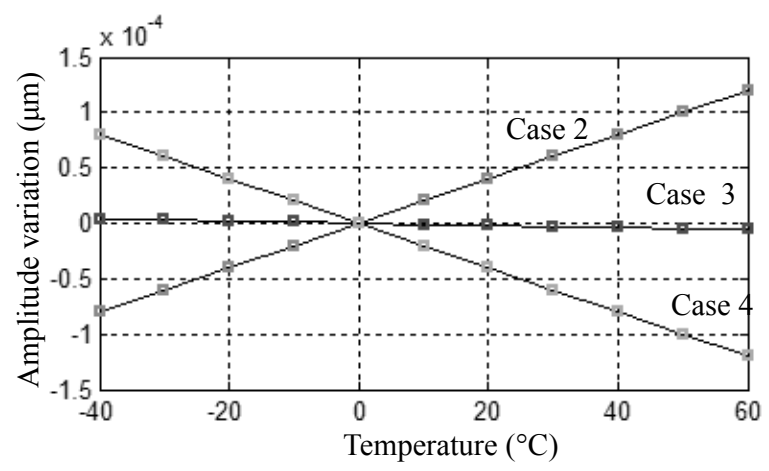

Fig. 8. Amplitude variation of the resonator. 


\section{Fabrication}

\subsection{Separate fabrication process of resonator}

A commercial precision fused silica cylinder is used for the cylinder wall of the combined resonator, whose purity is $99.99 \%$ and radius is $15 \mathrm{~mm}$, roundness is 0.01 $\mathrm{mm}$ (other geometric parameters are consistent with those listed in Table 2). In order to increase the strength of the glue between the cylinder wall and bottom disk, the surfaces of both sides of the fused silica cylinder were polished to a roughness of $2 \mu \mathrm{m}$ and a flatness of less than $5 \mu \mathrm{m}$. Figure 9 shows the fused silica cylinder wall after polishing.

The bottom disk of the combined resonator is made from a commercial precision fused silica wafer. Its thickness is less than $0.5 \mathrm{~mm}$ with a flatness error of $5 \mu \mathrm{m}$. Laser cutting technology is employed to machine the holes on the wafter. ${ }^{(16)}$ A UV laser dicing tool made by Suzhou Delphi Laser Corporation is used for wafer dicing. For the fused silica wafer, the power of the laser is $1.8 \mathrm{~W}$, and the rotary speed of the laser beam is about $6000 \mathrm{rpm}$. The fused silica wafer should be coated with a metallic film to form a conducting layer. Hence, a $\mathrm{Cr} / \mathrm{Au}$ film is deposited on the surface of the wafer by a vacuum coating system. Figure 10 shows the fabrication process of the fused silica wafer.

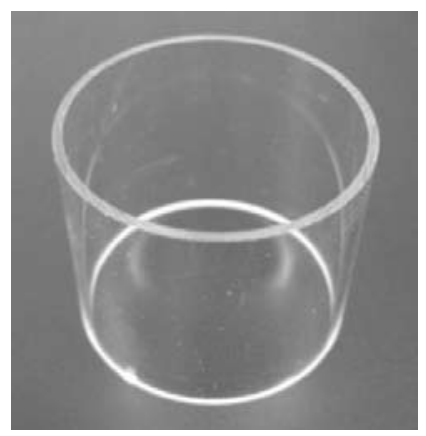

Fig. 9. Fused silica cylinder wall with polished ends.

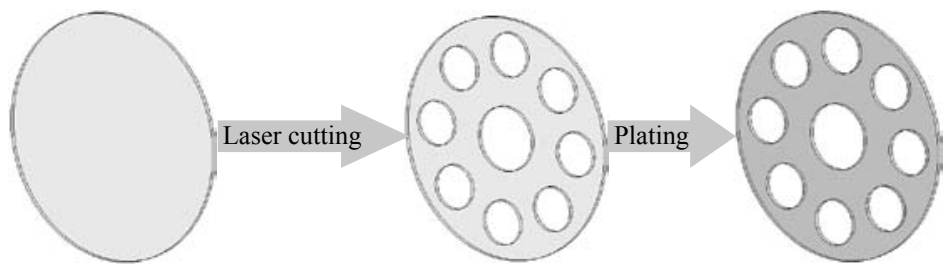

Fig. 10. Machining process of the fused silica wafer. 


\subsection{Combination of resonator}

After the cylinder wall and the bottom disk have been fabricated, both parts should be connected by glue. Considering the wide application of gluing technology in structure bonding, ${ }^{(17)}$ the method of gluing used to combine the gyroscope must be appropriate. It should be noted that the glue must be carefully chosen to obtain high thermal stability and long-term performance. The long-term behavior of glue depends on the type of glue and the environmental conditions. The composition of the glue used in this gyroscope includes epoxy resin, phenolic resin, and polyurethane.

The bonding process mainly consists of two steps: (1) The bottom disk is placed into a specially designed chuck, and the glue is added dropwise on the surface of the bottom disk where the connection will be made, as shown in Fig. 11(a). The chuck is a cylindrical shell whose inner diameter should be coincident with the outer diameter of the resonator. (2) The cylinder wall is placed into the chuck. Due to the fixing of the chucking, the cylinder wall and the bottom disk are strictly coaxial, as shown in Fig. 11(b). The glue is a fluid with low viscosity. Therefore, the small gap between the cylinder wall and the bottom disk can be completely filled with the glue easily. The curing of the glue is implemented at room temperature, and $6 \mathrm{~h}$ curing time is needed. After cleaning the shell surface, the bonding of the resonator was completed.

Finally, the piezoelectric electrodes were glued to the fused silica bottom disk. The piezoelectric electrodes should be separated from each other by exactly $45^{\circ}$, and the glue connecting the base plate and the electrodes should be electrically conductive and strong. The resonator was installed on a substrate, as shown in Fig. 12.

\subsection{Balancing of resonator}

The natural frequency split (the split of the two work mode frequencies) is mainly caused by fabrication errors of the resonator, which is the main quadrature error source of the gyroscope. ${ }^{(18,19)}$ Figure 13(a) shows the frequency response curves of the driving mode and sensing mode for the combined resonator before mechanical balancing where the natural frequency split is $0.35 \mathrm{~Hz}$. To sense the angular rate accurately, the natural frequency split of this symmetrical structure should be reduced to a sufficiently small magnitude. ${ }^{(20)}$ In order to minimize the natural frequency split, the mechanical balancing

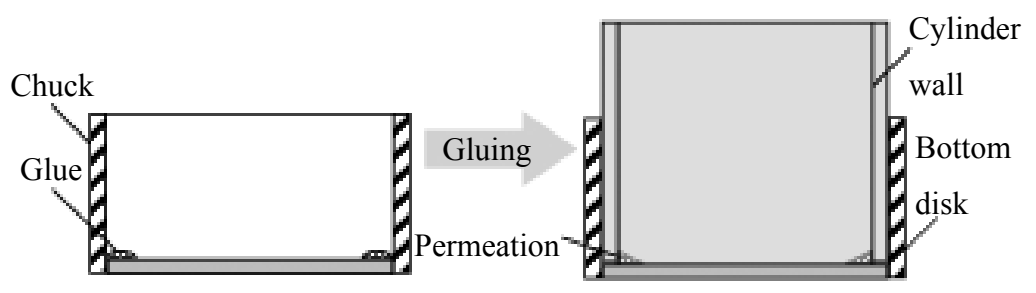

Fig. 11. Bonding of the resonator. (a) Drip the glue on the bottom disk and (b) connect the cylinder wall and bottom disk. 


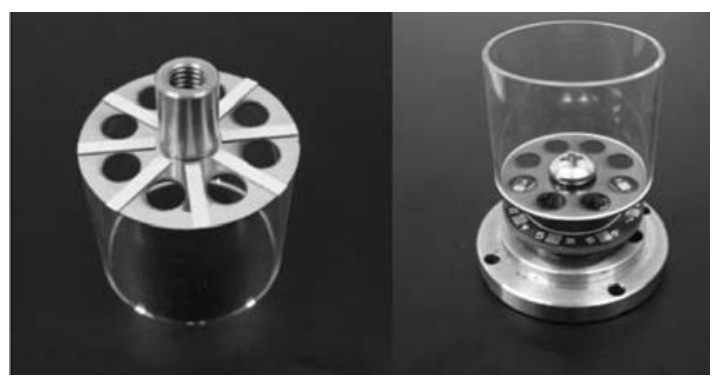

Fig. 12. Resonator of prototypal gyroscope.

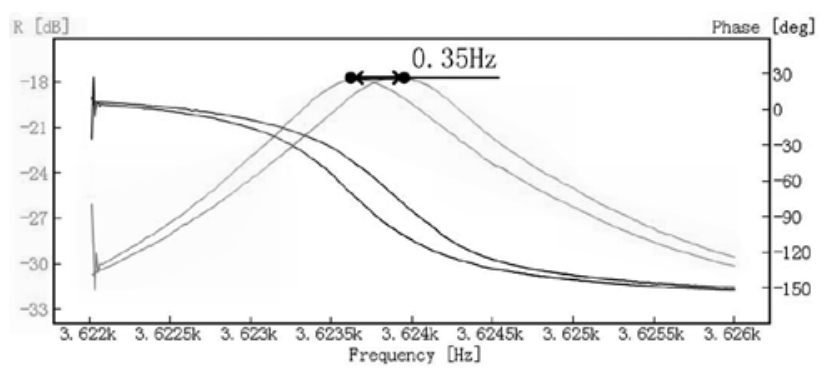

(a)

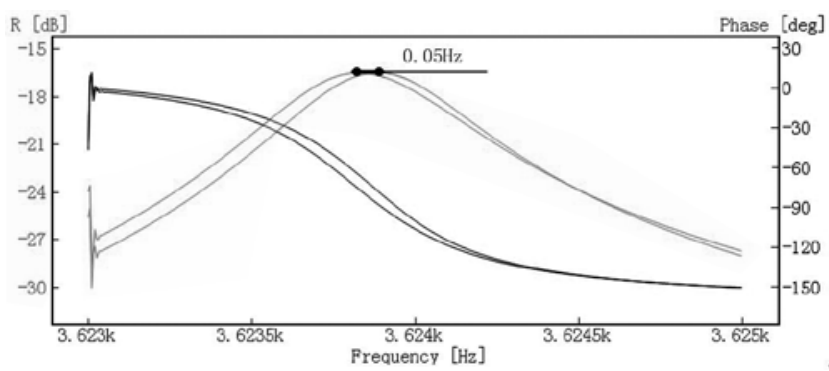

(b)

Fig. 13. Frequency response curves. (a) Before mechanical balancing and (b) after mechanical balancing.

of the resonator is necessary. The conventional mechanical balancing procedure generally involves the addition or removal of masses, ${ }^{(10)}$ which is an important process for improving the performance of a gyroscope. The balancing method has been well studied, and detailed information can be found in refs. 21 and 22 .

It is known that the driving mode and sensing mode of a resonator are always separated from each other by $45^{\circ}$. For an imperfect resonator, the two modes vibrate along the high-frequency and low-frequency axes of the resonator, respectively. Thus, 
the frequency split is the difference between the two frequencies. In this study, some tiny metal pieces are glued to the resonator to decrease the natural frequency split, as shown in Fig. 14. These additional masses are located on the high-frequency axis of the resonator at the open end of the cylinder wall. However, vibration may potentially be induced by the additional masses, which has been studied in refs. 18 and 19. Figure 13(b) shows the frequency response curves of the driving mode and sensing mode for the combined resonator after mechanical balancing, which reduced the natural frequency split to $0.05 \mathrm{~Hz}$.

\subsection{Control circuit}

The overall performance of the cylinder vibrating gyroscope not only depends on the structural precision; the control circuit also plays an important role. Figure 15 shows

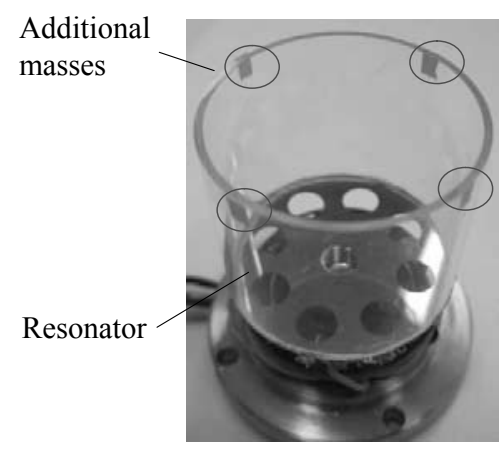

Fig. 14. Mechanical balancing by adding masses.

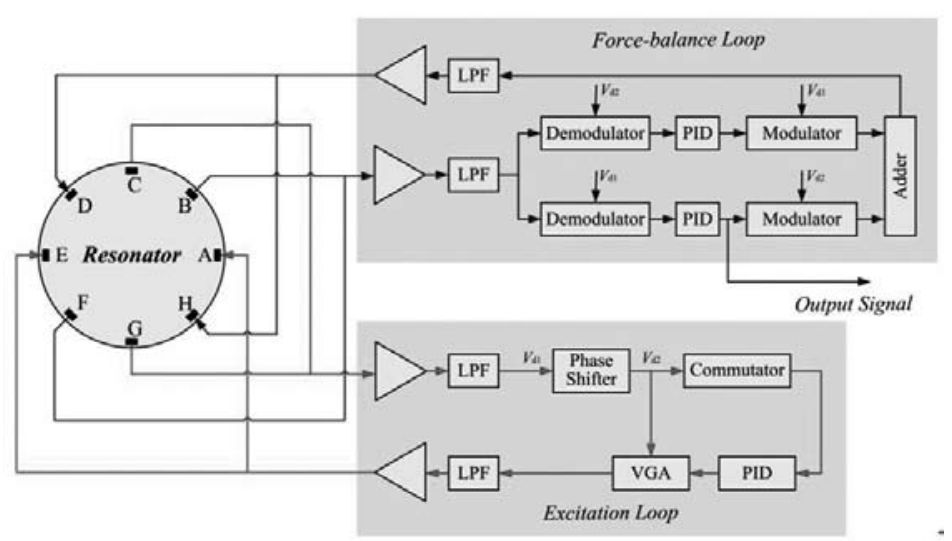

Fig. 15. Block diagram of the control method. 
a block diagram of the control method, which includes an excitation control loop and a force-balance control loop.

The excitation control loop excites the driving mode of the resonator. It keeps the output voltage of piezoelectric elements $\mathrm{A}$ and $\mathrm{E}$ at a constant value through the proportional integral derivative (PID) regulator. In this article, the $\pi / 2$ shifter is designed to satisfy the self-excited oscillation condition that phase delay of the excitation loop is $2 n \pi$. Piezoelectric elements $\mathrm{A}$ and $\mathrm{E}$ receive an activating voltage ( $5 \mathrm{~V}$ for example) and excite the active mode of the resonator; piezoelectric elements $\mathrm{C}$ and $\mathrm{G}$ output voltage proportional to the amplitude of the vibration to a voltage gain amplifier, which provides negative feedback control to stabilize the vibration amplitude of the resonator at a constant value to avoid the effect of temperature variation and power source instability during gyroscope operation.

The force-balance loop keeps the output voltage of piezoelectric elements $\mathrm{B}$ and $\mathrm{F}$ at zero through the PID regulator, which restrains the sensing mode of the resonator and improves the angular velocity response bandwidth of the gyroscope. When the resonator rotates about its central axis, the sense mode of the resonator is excited by Coriolis force, and the piezoelectric elements $\mathrm{B}$ and $\mathrm{F}$ output signal, and this signal is demodulated and modulated with signals from the excitation loop to form a compensation signal. The compensation signal is supplied to piezoelectric elements $\mathrm{D}$ and $\mathrm{H}$, which generate compensation forces to balance the Coriolis force and restrain the sense mode. Therefore, the resonator keeps on working in the active mode and has a high dynamic response bandwidth. The feedback signal from the output of the PID regulator is proportional to the input angular velocity.

\section{Gyroscope Test}

A test of the prototypal gyroscope is conducted, including a parameter test of the resonator and a performance test of the gyroscope.

\subsection{Qfactor test}

The $\mathrm{Q}$ factor is an important index for judging a gyroscope's dynamics and an important parameter contributing to a gyroscope's sensitivity. ${ }^{(23)}$ The harmonic response was measured by using an NF FRA5087 frequency response analyzer. An inducing voltage was applied on a pair of piezoelectric electrodes, and the response voltage was picked up from the piezoelectric electrodes located in the vertical direction. The resonating response is shown in Fig. 16. The Q factor of the prototypal gyroscope was measured. The test results showed that the $\mathrm{Q}$ factors of the gyroscope are 6250 and 6430 , respectively, at atmosphere pressure and in vacuum. Thus, vacuum packaging is not necessary for this gyroscope.

Since the cylinder wall and the bottom disk of the combined resonator are connected by glue, this causes energy loss and reduces the $\mathrm{Q}$ factor. In order to test the influence of the connecting glue on the $\mathrm{Q}$ factor, a monolithic fused silica resonator was employed for comparison, as shown in Fig. 17. The test result shows that the integral resonator's $Q$ factor is 7000 . Thus, the $\mathrm{Q}$ factor of the combined resonator is only slightly smaller than that of the monolithic resonator. 


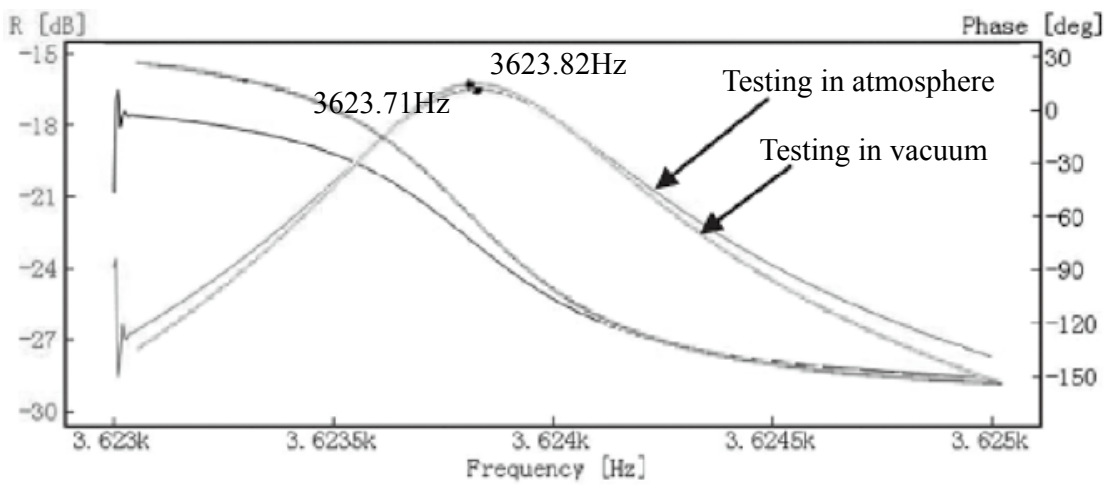

Fig. 16. Resonating response of the combined gyroscope.

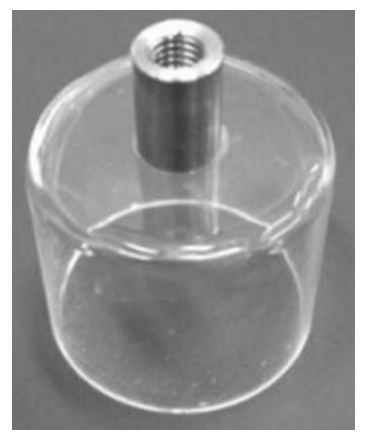

Fig. 17. Monolithic fused silica resonator.

\subsection{Scale factor and bias stability test}

A scale factor response measurement of the combined fused silica gyroscope was implemented, and Fig. 18 shows the output sensing voltages under different input angular velocities. It is calculated that the scale factor of the combined gyroscope is $110 \mathrm{mV} / \% / \mathrm{s}$.

Bias stability is the core parameter for gyroscope performance. For a cylinder shell vibrating gyroscope, zero bias is the output value of the gyroscope when the resonator is at rest. The drift of the prototypal gyroscope is recorded over $2 \mathrm{~h}$ at room temperature, as shown in Fig. 19. The Allan variance is used as a measure to assess the bias stability of the prototypal gyroscope. As shown in Fig. 20, the gyroscope's bias stability at room temperature is about $1.6^{\circ} / \mathrm{h}$. The result shows that the bias stability of the combined fused silica gyroscope has tactical-grade performance at a constant temperature.

\subsection{Temperature frequency factor test}

A preliminary temperature test for the resonator is also conducted in this study. It is considered that the stability of the eigenfrequency is closely related to the stability of the 


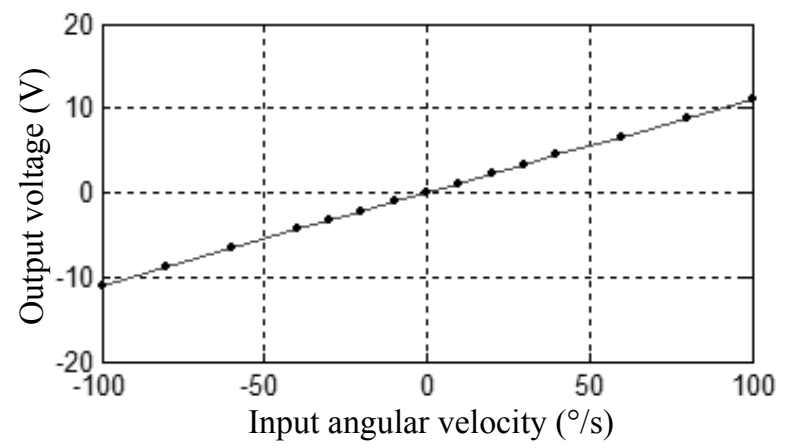

Fig. 18. Scale factor response measurement of the gyroscope.

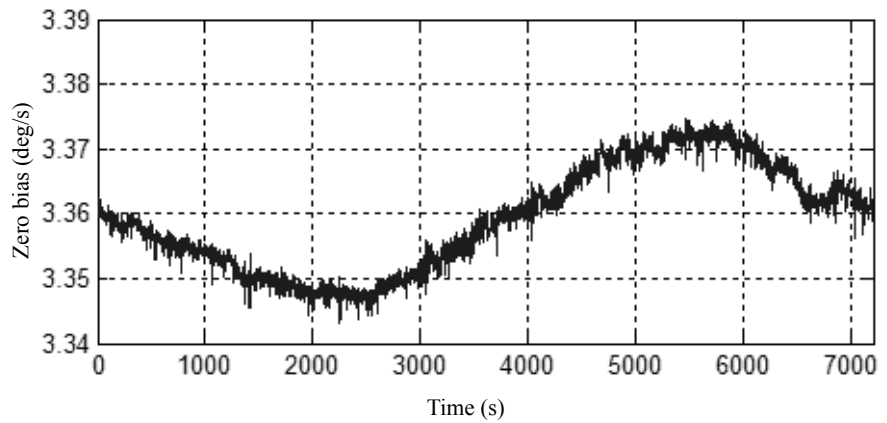

Fig. 19. Output voltage of the gyroscope over $2 \mathrm{~h}$.

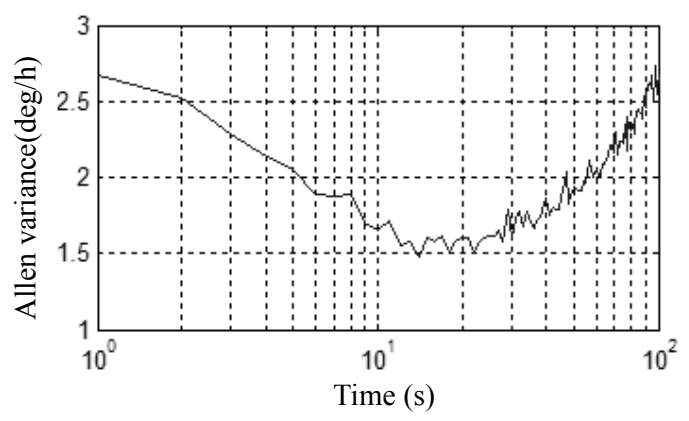

Fig. 20. Allan variance at room temperature. 
vibratory structure. The control circuit of the gyroscope will also benefit from a stable eigenfrequency. Thus, a frequency stability test of the resonator is implemented. The stability of the resonance frequency of the combined fused silica resonator is tested from -40 to $60{ }^{\circ} \mathrm{C}$, and the test data is shown in Fig. 21. For the sake of comparison, the test result of the monolithic resonator is also included.

The results show that the temperature frequency factor ${ }^{(24)}$ of the combined fused silica resonator is $8.64 \times 10^{-5} \% \mathrm{C}$, slightly larger than that of the monolithic fused silica resonator $\left(8.02 \times 10^{-5} \% \mathrm{C}\right)$. Therefore, the novel combined fused silica cylinder shell vibrating gyroscope may have the potential to maintain good stability under varying temperature.

\section{Conclusions}

This paper presents the simulation, fabrication, and performance test of a novel combined fused silica cylinder vibrating gyroscope. Two parts of the fused silica resonator, the cylinder wall and the bottom disk, are fabricated separately and then are connected by a special glue. The novel fabrication method can reduce the difficulty of manufacturing the fused silica cylinder vibrating gyroscope since the machining stress acting on the turning of the thin wall can be largely avoided. Simulation results show that the temperature stability of the resonator is still acceptable when the disadvantages of the combined method are taken into account. The whole manufacturing process is presented, including the end polishing, the structure bonding and the mass balancing. The radius of the resonator is $30 \mathrm{~mm}$, the height is $25 \mathrm{~mm}$ and the roundness is 10 $\mu \mathrm{m}$. Experimental tests of the prototypal gyroscope were conducted. A scale factor of $110 \mathrm{mV} / \% / \mathrm{s}$ is measured in a force-balance control loop. The experimental results also show that the combined fused silica cylinder vibrating gyroscope has a good bias stability, which can reach a tactical-grade level of $1.6^{\circ} / \mathrm{h}$. Since the tested gyroscope is just a preliminary prototype, its performance can be further improved by optimizing

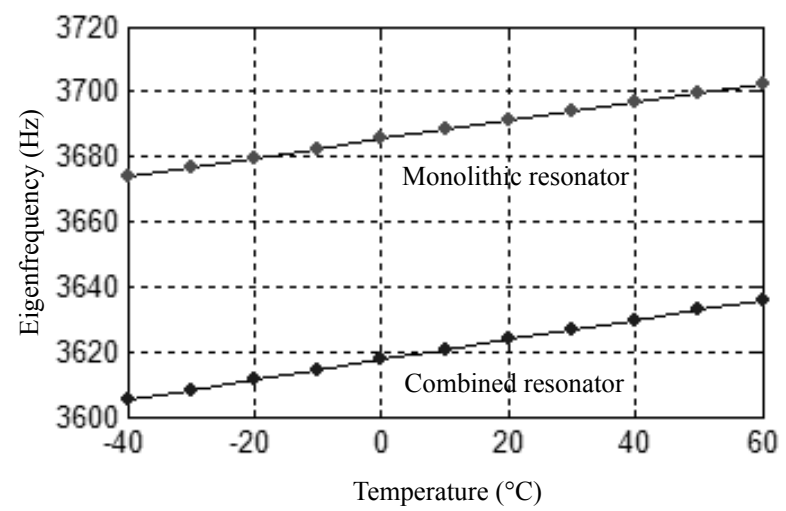

Fig. 21. Frequency-temperature curves of resonators. 
the resonator structure, improving the precision of the resonator, and employing a better connection method.

\section{Acknowledgements}

The authors would like to thank the Laboratory of Microsystems, National University of Defense Technology, China, for technical support and access to equipment. This work was supported by the National Natural Science Foundation of China (Grant Nos. 51005239 and 51275522).

\section{References}

1 A. M. Shkel: Aerosp. Electron. Syst. 7 (1992) 40.

2 V. A. Matveev, M. A. Basarab and A. V. Alekin: Solid State Wave Gyro (National Defense Industry Press, Beijing, 2009) p. 3.

3 V. Chikovani, E. Okon and A. Barbashov: Proc. IEEE/ION Position, Location and Navigation Symp, 2008, p. 238.

4 W. S. Watson: J. Micro Mach. 10 (2010) 171.

5 W. K. Sung, M. Dalal and F. Ayazi: IEEE 23rd Int. Conf. on IEEE Microelectromechanical Systems (MEMS), 2010, p. 116.

6 F. Ayazi and K. Najafi: IEEE J. Microelectromech. Syst. 10 (2001) 169.

7 J. S. Lyons and T. L. Starr: J. Am. Ceram. Soc. 776 (1994) 1673.

8 E. A. Izmailov, M. M. Kolesnik, A. M. Osipov and A. V. Akimov: Int. Conf. Integrated Navigation Systems, 1999, p. 24.

9 V. V. Chikovani, Yu. A. Yatsenko, and A. S. Barabashov: A\&E Syst. 5 (2009) 40.

10 P. W. Loveday: Analysis and Compensation of Imperfection Effects in Piezoelectric Vibratory Gyroscope (Virginia Polytechnic Institute and State University, Blacksburg, 1999) p. 24.

11 X. Xu and S. Yuan: Piezoelectrics \& Acoustooptics 6 (2006) 730.

12 A. K. Singh: Def. Sci. J. 57 (2007) 95.

13 D. D. Lynch: Proc. Symp. Gyro Technology, 1998, p. 1.0.

14 Y. Wu, X. Xi, Y. Tao, X. Wu and X. Wu: Sensors 11 (2011) 7665.

15 D. Liu and L. Xu: Piezoelectric Ferroelectric Material and Device (Huazhong University of Science and Technology Press, Wuhan, 1990) p. 123.

16 J. Liu, J. Lu, X. Ni, G. Dai, L. Zhang and Y. Chen: Opt. Appl. 1 (2011) 247.

17 T. Yasuhiro, N. Tsuyoshi, O. Takafumi and M. Zenichiro: J. Soc. Mater. Sci. 50 (2001) 994.

18 M. Putty: Ph.D. Thesis, The University of Michigan (1995).

19 S. Lee and K. Najafi: Sens. Actuators, A 171 (2011) 163.

20 A. K. Rourke, S. Mcwilliam and C. H. J Fox: J. Sound Vib. 248 (2001) 695.

21 P. Bisegna and G. Caruso: J. Sound Vib. 306 (2007) 691.

22 D. Schwartz, D. J. Kim and R. T. M'Closkey: Proc. American Control Conf. (2008) p. 3740.

23 J. Chihwan, S. Seonho, L. Byeungleul, K. Hyeonched and C. Kukjin: J. Micromech. Microeng. 14 (2004) 1530.

24 V. V. Chikovani, Y. A. Yatsenko, A. S. Barabashov, V. A. Kovalenko, V. I. Scherban and P. I. Marusyk: S. Petersburg conf. Integrated Navigation Systems, St., 2007, p. 74. 